EPJ Web of Conferences 37, 09029 (2012)

DOI: $10.1051 /$ epjconf/20123709029

(C) Owned by the authors, published by EDP Sciences, 2012

\title{
ABC Effect and Resonance Structure in the Double-Pionic Fusion to ${ }^{3} \mathrm{He}$
}

\author{
$\underline{\text { E. Perez del Rio }}^{1,2}$, M. Bashkanov ${ }^{1,2}$, H. Clement ${ }^{1,2, a}$, E. Doroshkevich ${ }^{1,2}$, A. Pricking ${ }^{1,2}$, \\ T. Skorodko ${ }^{1,2}$, and G. J. Wagner ${ }^{1,2}$ for the WASA-at-COSY Collaboration \\ 1 Physikalisches Institut, Univ. Tübingen, Auf der Morgenstelle 14, D-72076 Tübingen, Germany \\ 2 Kepler Center for Astro and Particle Physics, Univ. Tübingen, Auf der Morgenstelle 14, D-72076 \\ Tübingen
}

\begin{abstract}
Historically the double-pionic fusion to ${ }^{3} \mathrm{He}$ is the reaction, where the $\mathrm{ABC}$ effect (low-mass enhancement of the $\pi \pi$-invariant mass spectrum) was observed for the first time more than 50 years ago. Meanwhile exclusive and kinematically complete highstatistics measurements with WASA at COSY revealed a strict correlation between the $\mathrm{ABC}$ effect and a narrow resonance structure with $I\left(J^{P}\right)=0\left(3^{+}\right)$in the total cross section of the most basic double-pionic fusion, the $p n \rightarrow d \pi^{0} \pi^{0}$ reaction. In order to investigate the situation in the double-pionic fusion to ${ }^{3} \mathrm{He}$ the energy dependence of the $\mathrm{ABC}$ effect in the fusion to ${ }^{3} \mathrm{He}$ has been measured by fixed energy measurements of the type $p d \rightarrow{ }^{3} \mathrm{He} \pi \pi$ as well as by quasifree and coherent measurements of the type $d d \rightarrow{ }^{3} \mathrm{He} \mathrm{n}$ $\pi \pi$.
\end{abstract}

\section{Introduction}

The ABC effect - an intriguing low-mass enhancement in the $\pi \pi$ invariant mass spectrum - is known from inclusive measurements of two-pion production in nuclear fusion reactions to the few-body systems d, ${ }^{3} \mathrm{He}$ and ${ }^{4} \mathrm{He}$. It was first observed 1960 by Abashian, Booth and Crowe in the inclusive $p d \rightarrow{ }^{3} \mathrm{He} \mathrm{X}$ reaction [1]. Its explanation has been a puzzle since then. Therefore it has been named just after the initials of those authors. In subsequent bubble-chamber [2,3] and single-arm magnetic spectrometer measurements [4-11] this enhancement showed up in double-pionic fusion reactions leading to $d,{ }^{3} \mathrm{He}$ and ${ }^{4} \mathrm{He}$, if an isoscalar pion pair was produced. However, such an enhancement was not observed in fusion reactions leading to deuteron and triton, if an isovector pion pair was produced. These results led to the conclusion that this effect only appears in reactions, where the participating nucleons fuse to a nuclear bound system in the final state in combination with the production of an isoscalar pion pair.

In recent exclusive and kinematically complete measurement of the $p n \rightarrow d \pi^{0} \pi^{0}$ reaction it has been demonstrated [12-15] that the ABC effect in this basic double-pionic fusion reaction is correlated with a narrow structure in the total cross section with quantum numbers $I\left(J^{P}\right)=0\left(3^{+}\right)$, a mass of 2.37 $\mathrm{GeV}$ and a width of about $70 \mathrm{MeV}$. The mass is about $90 \mathrm{MeV}$ below $2 m_{\Delta}$, the mass of a $\Delta \Delta$ system and the width is three times narrower than expected from a conventional $t$-channel $\Delta \Delta$ process.

On the contrary the basic isovector fusion process $p p \rightarrow d \pi^{+} \pi^{0}$ exhibits neither an ABC effect nor a narrow resonance structure [14-16] in agreement with the observations with all other two-pion channels in $p p$ initiated reactions [17-20].

\footnotetext{
a e-mail: clement@pit.physik.uni-tuebingen.de
}

This is an Open Access article distributed under the terms of the Creative Commons Attribution License 2.0, which permits unrestricted use, distribution, and reproduction in any medium, provided the original work is properly cited. 


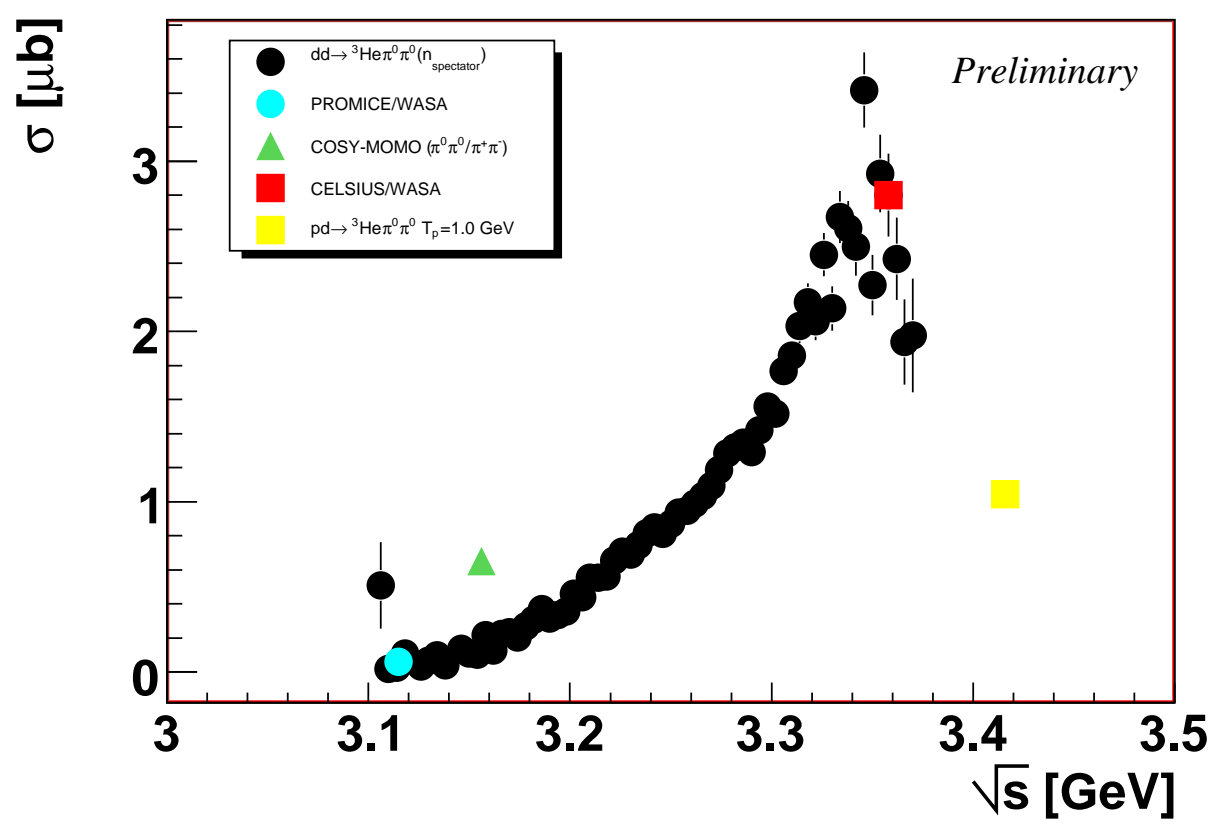

Fig. 1. Preliminary results for the total cross section for the double-pionic fusion to ${ }^{3} \mathrm{He}$ obtained from this experiment on $p d \rightarrow{ }^{3} \mathrm{He} \pi^{0} \pi^{0}$ at $T_{p}=1.0 \mathrm{GeV}$ (yellow square) and $d d \rightarrow{ }^{3} \mathrm{He} \pi^{0} \pi^{0}+n_{\text {spectator }}$ for the beam energy $T_{d}=1.4 \mathrm{GeV}$ (black dots) in comparison with previous results from PROMICE/WASA [25], CELSIUS/WASA [24] and COSY-MOMO [26].

\section{Experiment and Results}

In an effort to solve this long-standing puzzle by exclusive and kinematically complete high-statistics experiments, we have measured the fusion reactions to $\mathrm{d}[12,14],{ }^{3} \mathrm{He}$ and ${ }^{4} \mathrm{He}[15,21]$ with WASA at COSY $[22,23]$. Here we report on the measurements of the double-pionic fusion reaction $p d \rightarrow{ }^{3} \mathrm{He}$ $\pi^{0} \pi^{0}$. These measurements have been carried out either by a direct measurement at $T_{p}=1.0 \mathrm{GeV}$ (yellow square symbol in Fig. 1) as well as by utilizing quasi-free and coherent modes in $d d$ collisions at $T_{d}=1.4 \mathrm{GeV}$ (black solid dots in Fig. 1) and $1.7 \mathrm{GeV}$. That way these measurements cover the full energy region, where the $\mathrm{ABC}$ effect has been observed previously in inclusive reactions [1-3].

From a previous exclusive experiment at CELSIUS-WASA at $T_{p}=0.893 \mathrm{GeV}$ (red square symbol in Fig. 1) [24] it is known, that also the double-pionic fusion to ${ }^{3} \mathrm{He}$ exhibits a pronounced $\mathrm{ABC}$ effect. From the new data from COSY we see that this energy is just at about the cross section maximum. The data analysis is still in progress. As a preliminary result we show the energy dependence of the total cross section in Fig.1, where also the results from previous experiments at CELSIUS [13,25] and COSY [26] are plotted.

\section{Acknowledgments}

We acknowledge valuable discussions with C. Hanhart, E. Oset, A. Sibirtsev and C. Wilkin on this issue. This work has been supported by BMBF (06TU9193) and Forschungszentrum Jülich (COSYFFE). 


\section{References}

1. N. E. Booth, A. Abashian, K. M. Crowe, Phys. Rev. Lett. 7 (1961) 35; 5 (1960) 258; Phys. Rev. 132 (1963) $2296 \mathrm{ff}$

2. I. Bar-Nir et al., Nucl. Phys. B 54 (1973) 17

3. A. Abdivaliev et al., Sov. J. Nucl. Phys. 29(1979) 796

4. R. J. Homer et al., Phys. Rev. Lett. 9 (1964) 72

5. J. H.Hall et al., Nucl. Phys. B 12 (1969) 573

6. J. Banaigs et al., Nucl. Phys. B 67 (1973) 1

7. J. Banaigs et al., Nucl. Phys. B 105 (1976) 52

8. F.Plouin et al., Nucl. Phys. A 302 (1978) 413

9. F. Plouin, P. Fleury, C. Wilkin, Phys. Rev. Lett. 65 (1990) 690

10. R. Wurzinger et al., Phys. Lett. B 445 (1999) 423

11. for a review see A. Codino and F. Plouin, $\mathrm{LNS} / \mathrm{Ph} / 94-06$

12. P. Adlarson et al., Phys. Rev. Lett. 106, (2011) 202302

13. M. Bashkanov et al., Phys. Rev. Lett. 102 (2009) 052301; arXiv: 0806.4942 [nucl-ex]

14. M. Bashkanov et al., contribution to this conference

15. A. Pricking et al., contribution to this conference

16. F. Kren et al., Phys. Lett. B 684, (2010) 110 and B 702 (2011) 312; arXiv:0910.0995v2 [nucl-ex]

17. T. Skorodko et al., contribution to this conference

18. T. Skorodko et al., Phys. Lett. B 695, (2011) 115

19. T. Skorodko et al., Phys. Lett. B679 (2009) 30; arXiv: 0906.3087 [nucl-ex]

20. T. Skorodko et al., Eur. Phys. J. A 47, (2011) 108

21. A. Pricking, $\mathrm{PhD}$ thesis, Univ. Tübingen 2011; http://tobias-lib.uni-tuebingen.de/volltexte/2011/5695/pdf/ThesisFinal.pdf

22. Ch. Bargholtz et al., Nucl. Instrum. Methods A 547, (2005) 294

23. H. H. Adam et al., arXiv:nucl-ex/0411038, (2004)

24. M. Bashkanov et al., Phys. Lett. B 637 (2006) 223.

25. M. Andersson et al., Phys. Lett. B 485 (2000) 327

26. F. Bellemann et al., Phys. Rev. C 60 (1999) 061002 